\title{
CONTRIBUTION OF NON-TROGLOBIOTIC TERRESTRIAL INVERTEBRATES TO CARBON INPUT IN HYPOGEAN HABITATS
}

\author{
PRISPEVEK PREZIMUJOČIH NETROGLOBIONTSKIH \\ KOPENSKIH NEVRETENČARJEV K VNOSU OGLJIKA \\ V PODZEMELJSKE HABITATE
}

\author{
Tone NOVAK ${ }^{1}$, Franc JANŽEKOVIČ ${ }^{1}$ \& Saška LIPOVŠEK ${ }^{1,2}$
}

\begin{abstract}
UD 592:546.26:551.44 C

Tone Novak, Franc Janžekovič \& Saška Lipovšek: Contribution of non-troglobiotic terrestrial invertebrates to carbon input in hypogean habitats

Eleven of the most important terrestrial invertebrate species in Slovenian caves were analyzed for differences in their fresh and dry biomass, energy content and carbon bulk during winter. These data were combined with the species abundance in 54 caves and adits in order to estimate their organic carbon bulk and carbon input into these habitats. In Central European caves, Troglophilus cavicola, T. neglectus, Faustina illyrica, Amilenus aurantiacus and Scoliopteryx libatrix are the most important vectors of carbon between the epigean and hypogean habitats. In contrast to the general assumption, carbon total contribution to caves via dead bodies is only $0.15 \%$ of total migratory biomass, and it is not directly available to troglobionts because of infection with entomopathogenic fungi. In winter, together with predated migratory specimens, carbon input does not exceed $0.3 \%$ of the total migratory biomass. This situation should be carefully examined in every karstic region inhabited by distinctive fauna.
\end{abstract}

Keywords: biomass, carbon flux, caves, non-troglobiotic species, organic carbon, respiration.
Izvleček

UDK 592:546.26:551.44

Tone Novak, Franc Janžekovič ¿ Saška Lipovšek: Prispevek prezimujočih netroglobiontskih kopenskih nevretenčarjev $k$ vnosu ogljika vodzemeljske habitate

$\mathrm{V}$ enajstih najpomembnejših vrstah kopenskih nevretenčarjev $\mathrm{v}$ jamah Slovenije smo proučili razlike v biomasi, suhi masi, energijski vsebnosti in količini ogljika $\mathrm{v}$ zimskem obdobju. Te podatke smo obravnavali $\mathrm{v}$ kombinaciji $\mathrm{z}$ abundanco $\mathrm{v} 54$ jamah in rudnikih, da bi ocenili količino organskega ogljika $\mathrm{v}$ njih ter vnos ogljika v te habitate. V srednji Evropi so najpomembnejše vrste, ki vnašajo ogljik v podzemlje, Troglophilus cavicola, T. neglectus, Faustina illyrica, Amilenus aurantiacus in Scoliopteryx libatrix. V nasprotju s splošno domnevo, prispevajo poginuli osebki le $0,15 \%$ C glede na skupno migrirajočo biomaso, ki ga troglobionti ne morejo neposredno uporabiti zaradi okužb z entomopatogenimi glivami. Skupaj z uplenjenimi osebki vnos ne presega $0,3 \%$ biomase teh vrst. Te razmere je treba skrbno proučiti v vsakem kraškem predelu, kjer se migrirajoča favna razlikuje od ostalih območij.

Ključne besede: biomasa, dihanje, jame, tok ogljika, netroglobiontske vrste, organski ogljik.

\section{INTRODUCTION}

Maintenance of life, manifested through metabolic processes, is directly involved in carbon cycling, a substantial domain for biological and ecological studies. In the last two decades, biosequestration, the capture and storage of atmospheric $\mathrm{CO}_{2}$ by biotic processes and systems, has at- tracted much attention. This is because biosequestration is closely related to global warming, which currently represents one of the main threats to the Earth's biota, humans included, and which is caused in a large part by anthropogenic $\mathrm{CO}_{2}$ emissions (U.S. DOE 2008; Houghton

${ }^{1}$ Department of Biology, Faculty of Natural Sciences and Mathematics, University of Maribor, Koroška cesta 160, SI-2000 Maribor, Slovenia, e-mail: tone.novak@uni-mb.si; franc.janzekovic@uni-mb.si

${ }^{2}$ Medical Faculty, University of Maribor, Slomškov trg 15, SI-2000 Maribor, Slovenia, e-mail: sasa.lipovsek@uni-mb.si

Received/Prejeto: 1.2.2013 
2009; Riebeek 2011). The natural carbon cycle includes carbon flow through inorganic and organic compounds, i. e., through abiotic and biotic systems, although our understanding of changes in terrestrial biomass in this respect remains rather rudimentary (Perruchoud et al. 2000; Houghton et al. 2009). Soil organic carbon is the main global carbon stock in recent carbon cycling; more than half of cycling carbon is currently stored in soils (Perruchoud et al. 2000; Schmidt et al. 2011).

In hypogean habitats, in the absence of photosynthesis, carbon compounds are fewer and less diverse in comparison to epigean habitats (Culver \& Pipan 2009). So far, chemoautotrophy has been confirmed in only a very few caves (Sârbu et al. 1996, Engel 2005, Por 2007). The diversity and abundance of the biota in most other, heterotrophic hypogean habitats depend on allochthonous energy resources. These habitats, cave streams for example, are more likely to be carbon - rather than nutrient-limited (Simon \& Benfield 2002). Among heterotrophic organisms in these habitats, the flow of carbon includes the uptake of substrates rich in organic carbon and other nutrients as well as their prey, their metabolism and incorporation of nutrients into the body, and a permanent, gradual loss of carbon from the body through elimination and respiration.

Organic matter is conveyed into the subterranean environment by water, gravity, wind or active movements of organisms. Water and soil are the main abiotic vectors supplying the subterranean area with food, whilst growing roots and migrating animals are the most important biotic resources (Tercafs 2001; Culver \& Pipan 2009; Fong 2011). In addition, guano supports a specific subterranean community, the guanobionts (Deharveng \& Bedos 2000; Gnaspini 2005). Food takes the form of dissolved organic carbon (DOC), fine (FPOM) and coarse particulate organic matter (CPOM), eggs, cadavers, excrement and predated specimens. A conspicu- ous amount of organic carbon is incorporated in nontroglobiotic animals (i. e., trogloxenes and troglophiles; see Novak et al. 2012 for comments) that move daily or seasonally between surface and subterranean habitats and supply food directly to the subterranean communities (Fong 2011).

Careful accounting of inputs and outputs to a karst basin and measures of standing stocks and fluxes (transport and respiration) within basins are required to improve our knowledge of the carbon cycle within karstic ecosystems (Simon et al. 2007). From this perspective, the contribution of terrestrial trogloxenic species, species that periodically leave the cave, to subterranean communities has been underestimated (Fong 2011) and understudied. Till recently, most such studies have involved estimating the amount of excrement/guano or cadavers or eggs laid in caves (e. g., Jequier 1964; Taylor et al. 2005). Novak \& Kuštor (1982) published the first measurements of the energy content in selected nontroglobiotic species.

In dormancy, metabolism slows down considerably, and development and reproduction are suppressed (Denlinger 2002). This period is then appropriate for estimating energy requirements in animals maintaining basal metabolism, i. e., the minimal loss of organic carbon via respiration. Some non-troglomorphic terrestrial animals have been studied for their energy metabolism during overwintering (Lipovšek et al. 2004, 2008, 2009, 2011; Novak et al. 2004).

In this contribution, our goal is to estimate how these taxa are involved in carbon flow with respect to their contribution of organic carbon to hypogean habitats, and what is the carbon lost via respiration during their winter sojourn in these habitats. We hypothesized that more abundant species and those with larger masses are more important in this respect.

\section{MATERIALS AND METHODS}

For this study, we combined two data sets. The first is a field data set on the more abundant invertebrates (listed in Novak et al. 2012) from 51 caves and three abandoned mines in central and northern Slovenia (listed and mapped in Novak 2005; in the following: caves). The second data set includes biometric, biochemical and cytological data provided by a separate study of 11 of the more abundant non-troglomorphic invertebrate species which inhabit these caves during winter or which are important for their large mass (Tab. 1). Fresh mass was used in calculating their biomass bulk, and their dry mass to assess their carbon bulk and loss during winter. The caves were investigated between 1977 and 2001. We sampled in January, April, July and October in a total of 617 sampling sections, every $3.5 \mathrm{~m}$, on average. Faunal records were provided on two visits within 45-48 hrs by observing the cave walls, ceiling and floor and applying standardized, baited pit-fall trapping and Berlese funnels. Altogether, 2468 records were provided, referring to 173,008 individuals of 600 estimated species in 
total (details in Novak et al. 2004; Novak 2005). During winter, numbers of individuals in all the species varied by ca. $5 \%$ to $40 \%$, mostly because of migration between the caves and adjacent habitats inaccessible to humans; this variation is not part of the present study. In order to avoid these disturbances, we estimated the biochemical changes as if the abundances did not change during overwintering. Data on dead organisms and excrement were estimated because the study was not designed to monitor these. Predated individuals are discussed in Novak et al. (2010). By 2013, 64 caves in total had been investigated in the same way (own unpublished data). In four of these monthly monitoring provided important supporting information.

Specimens for biometrical, biochemical and cytological study were collected between 2003 and 2011 from seven caves in Slovenia (locality centroid 46 $24^{\prime} 55^{\prime \prime}$

Tab. 1: Species investigated for their participation in carbon and energy fluxes in subterranean environments in winter.

\begin{tabular}{l|l|l}
\hline Higher taxon & Family & Species \\
\hline Gastropoda & Helicidae & Chilostoma (Josephinella) lefeburiana (Férussac, 1821) \\
\hline & & Faustina illyrica (Stabile, 1864) \\
\hline Opiliones & Phalangiidae & Amilenus aurantiacus (Simon, 1881) \\
\hline & & Gyas annulatus (Olivier, 1791) \\
\hline & & Gyas titanus Simon, 1879 \\
\hline Araneae & Tetragnathidae & Meta menardi (Latreille, 1804) \\
\hline Coleoptera & Carabidae & Laemostenus schreibersii (Küster, 1846) \\
\hline & Geometridae & Triphosa dubitata (Linnaeus, 1758) \\
\hline Orthoptera & Noctuidae & Scoliopteryx libatrix (Linnaeus, 1758) \\
\hline & & Troglophilus cavicola (Kollar, 1833) \\
\hline & & Troglophilus neglectus Krauss, 1879
\end{tabular}

$\mathrm{N}, 15^{\circ} 10^{\prime} 31^{\prime \prime}$ E, altitude $600-740 \mathrm{~m}$ ), four among them from the list of 54 caves, and from a cave in northeastern Italy $\left(45^{\circ} 50^{\prime} 04^{\prime \prime} \mathrm{N}, 13^{\circ} 34^{\prime} 54^{\prime \prime} \mathrm{E}\right)$. Four among these caves were from the list of 54 caves. Specimens of each stage and sex-depending on the species-were measured for their size, fresh and dry mass, and lipid, glycogen and water content at the beginning (November), the middle (January) and the end of overwintering (March). Three to five additional specimens were analyzed histologically and cytologically in the control. For these analyses, specimens were killed by exposure for two hours to $-20^{\circ} \mathrm{C}$. The dry mass was determined after 15 days of vacuum desiccation under $\mathrm{P}_{2} \mathrm{O}_{5}$ (details in Lipovšek et al. 2004, 2008, 2009, 2011; Novak et al. 2004). Invertebrate dry mass is ca. $50 \%$ carbon, on average (Salonen et al. 1976), we therefore estimated carbon content as half the dry mass, and the carbon loss as $50 \%$ the dry mass lost during overwintering. The energetic input was calculated upon the biomass and energetic data in selected species-seven among those from the 11-species-list (Novak \& Kuštor 1982) (Tab. 4).

T-tests were used to identify significant differences in total fresh and dry mass between the beginning and the end of overwintering. The data analysis was carried out with the statistical software SPSS 19.0.

\section{RESULTS}

Table 2 shows individual fresh mass in the 10 species from the 54 caves plus Chilostoma from a cave in Italy and t-test between the series at the beginning and at the end of overwintering; their dry mass is presented in Tab. 3. Estimated average individual energy budgets are shown in Tab. 4. The selected species demonstrate various types of life cycles with respect to active or inactive life in hypogean habitats (active state, quiescence, diapause), strategies and the span of dormancy (Tab. 5), and strategies and level of efficiency in using energy-supplying compounds. Numbers of dead individuals and their dead biomass are also presented in Tab. 5. Fresh mass increased in most species overwintering deeper inside caves. Given this finding, the measurements for T. neglectus should be repeated on account of their discrepancy.
Both snails and both butterflies most probably decreased their fresh mass, owing to the diminished water content, due to a drop in relative humidity in the cave entrance sections. Both Troglophilus moult during overwintering (Novak \& Kuštor 1983), but T. cavicola larvae had obviously fed before sampling at the end of overwintering, probably because the sampling date was possibly past the end of winter. The same was true in F. illyrica.

After overwintering, the total fresh mass of these 11 species increased by $6.71 \%$, while their dry mass decreased by $10.09 \%$, and energy by $10.57 \%$. Their total organic carbon was $474.49 \mathrm{~g}$ at the beginning, and its respiration loss was $47.86 \mathrm{~g}$ by the end of winter. Their total dry necromass contributed $5.59 \mathrm{~g}$ of organic carbon to the caves, and yielded $1.07 / 0.93 \%$ of the fresh mass at 
Tab. 2: Individual fresh mass in the 11 species under investigation. $t$-test between the series at the beginning and at the end of overwintering. Significant p-values are in bold.

\begin{tabular}{|c|c|c|c|c|c|}
\hline $\begin{array}{c}\text { Species } \\
\text { N of individuals; } \\
\text { beginning-end } \\
\text { of overwintering }\end{array}$ & $\begin{array}{c}\text { Beginning } \\
\text { Mean } \pm S D \\
\text { Min-Max } \\
{[\mathrm{mg}]}\end{array}$ & $\begin{array}{c}\text { End } \\
\text { Mean } \pm S D \\
\text { Min-Max } \\
{[\mathrm{mg}]}\end{array}$ & $\begin{array}{c}\text { Average } \\
\text { difference } \\
\text { beginning-end } \\
\text { of over-wintering [\%] }\end{array}$ & $t ; p$ & $d f$ \\
\hline $\begin{array}{l}\text { F. illyrica } \\
5-5 \\
\end{array}$ & $\begin{array}{c}1944.3 \pm 43.6 \\
1910.9-2019.8 \\
\end{array}$ & $\begin{array}{c}1672.5 \pm 77.2 \\
1546.1-1757.5 \\
\end{array}$ & -13.98 & $\begin{array}{c}6.50 \\
<\mathbf{0 . 0 0 1} \\
\end{array}$ & 8 \\
\hline $\begin{array}{c}\text { C. lefeburiana } \\
5-6 \\
\end{array}$ & $\begin{array}{c}2202.4 \pm 253.7 \\
1901.1-2575.5 \\
\end{array}$ & $\begin{array}{c}1582.6 \pm 376.4 \\
1209-2199.8 \\
\end{array}$ & -28.14 & $\begin{array}{c}3.11 \\
\mathbf{0 . 0 1 3} \\
\end{array}$ & 9 \\
\hline $\begin{array}{c}\text { A. aurantiacus } \\
5-5\end{array}$ & $\begin{array}{r}14.7 \pm 1.6 \\
12.2-16.3\end{array}$ & $\begin{array}{c}16.1 \pm 0.1 \\
15.2-17.4 \\
\end{array}$ & 9.52 & $\begin{array}{c}1.70 \\
0.128\end{array}$ & 8 \\
\hline $\begin{array}{c}\text { A. aurantiacus }+9 \\
5-5\end{array}$ & $\begin{array}{l}20.3 \pm 2.5 \\
17.5-24.1\end{array}$ & $\begin{array}{l}22.2 \pm 2.2 \\
19.124 .9\end{array}$ & 9.36 & $\begin{array}{l}1.280 \\
0.236 \\
\end{array}$ & 8 \\
\hline $\begin{array}{c}\text { G. annulatus } \\
10-9\end{array}$ & $\begin{array}{c}29.89 \pm 5.29 \\
22.5-38.2\end{array}$ & $\begin{array}{c}30.72 \pm 4.24 \\
26.2-38.7\end{array}$ & 2.78 & $\begin{array}{c}0.38 \\
0.712 \\
\end{array}$ & 17 \\
\hline $\begin{array}{c}\text { G. titanus } \\
21-24 \\
\end{array}$ & $\begin{array}{c}42.9 \pm 8.13 \\
32-58.7 \\
\end{array}$ & $\begin{array}{l}44.8 \pm 12.0 \\
29.8-75.3 \\
\end{array}$ & 4.43 & $\begin{array}{l}0.43 \\
0.667 \\
\end{array}$ & 43 \\
\hline $\begin{array}{l}\text { M. menardi } \\
10-12 \\
\end{array}$ & $\begin{array}{c}248.2 \pm 80.2 \\
164.8-377.8 \\
\end{array}$ & $\begin{array}{c}290.6 \pm 89.5 \\
171.6-432.2 \\
\end{array}$ & 17.08 & $\begin{array}{l}1.13 \\
0.271 \\
\end{array}$ & 20 \\
\hline $\begin{array}{c}\text { L. schreibersii } \\
5-10\end{array}$ & $\begin{array}{l}61.8 \pm 12.2 \\
49.7-79.4 \\
\end{array}$ & $\begin{array}{l}60.6 \pm 19.4 \\
36.3-94.1 \\
\end{array}$ & -1.94 & $\begin{array}{l}0.32 \\
0.755 \\
\end{array}$ & 13 \\
\hline $\begin{array}{c}\text { T. dubitata } \\
10-11\end{array}$ & $\begin{array}{l}58.8 \pm 7.6 \\
46.6-72.4 \\
\end{array}$ & $\begin{array}{c}50.6 \pm 8.4 \\
39.2-63.6 \\
\end{array}$ & -13.95 & $\begin{array}{l}2.39 \\
\mathbf{0 . 0 2 8} \\
\end{array}$ & 19 \\
\hline $\begin{array}{c}\text { S. libatrix } \\
10-10\end{array}$ & $\begin{array}{c}261.1 \pm 33.6 \\
223.4-312.4 \\
\end{array}$ & $\begin{array}{c}228.8 \pm 23.7 \\
182.5-260.4\end{array}$ & -12.37 & $\begin{array}{l}2.34 \\
\mathbf{0 . 0 3 1}\end{array}$ & 18 \\
\hline $\begin{array}{c}\text { T. cavicola } \\
6-5\end{array}$ & $\begin{array}{c}477.8 \pm 51.2 \\
402.1-538.4 \\
\end{array}$ & $\begin{array}{c}596.1 \pm 47.1 \\
521-643.2 \\
\end{array}$ & 24.76 & $\begin{array}{l}3.76 \\
\mathbf{0 . 0 0 5}\end{array}$ & 9 \\
\hline $\begin{array}{c}\text { T. cavicola } \text { ㅇ } \\
5-5\end{array}$ & $\begin{array}{c}541.2 \pm 50.3 \\
504.1-629.4 \\
\end{array}$ & $\begin{array}{c}611.7 \pm 52.8 \\
534.9-658.4 \\
\end{array}$ & 13.03 & $\begin{array}{l}2.19 \\
0.060 \\
\end{array}$ & 8 \\
\hline $\begin{array}{c}\text { T. cavicola larvae } \\
10-10 \\
\end{array}$ & $\begin{array}{c}87.1 \pm 7.3 \\
77.5-99.2 \\
\end{array}$ & $\begin{array}{c}149.3 \pm 26.6 \\
101.4-183.9 \\
\end{array}$ & 71.41 & $\begin{array}{c}7.96 \\
<\mathbf{0 . 0 0 1} \\
\end{array}$ & 18 \\
\hline $\begin{array}{c}\text { T. neglectus } \\
4-2\end{array}$ & $\begin{array}{c}504.7 \pm 69.9 \\
407.7-557.3 \\
\end{array}$ & $\begin{array}{l}442.5 \pm 165.7 \\
325.3-559.7 \\
\end{array}$ & -12.32 & $\begin{array}{c}0.81 \\
\mathbf{0 . 0 4 7} \\
\end{array}$ & 4 \\
\hline $\begin{array}{c}\text { T. neglectus } q \\
5-6\end{array}$ & $\begin{array}{l}649.3 \pm 145.0 \\
428.4-788.7 \\
\end{array}$ & $\begin{array}{c}350.8 \pm 191.8 \\
71.4-672.8 \\
\end{array}$ & -45.97 & $\begin{array}{l}2.20 \\
0.056 \\
\end{array}$ & 9 \\
\hline $\begin{array}{c}\text { T. neglectus larvae } \\
13-14\end{array}$ & $\begin{array}{c}133.3 \pm 101.7 \\
48.6-383.6 \\
\end{array}$ & $\begin{array}{c}149.0 \pm 115.3 \\
73.6-441.9 \\
\end{array}$ & 11.78 & $\begin{array}{l}0.56 \\
0.565 \\
\end{array}$ & 25 \\
\hline
\end{tabular}

the beginning/the end of overwintering, and 3.43/3.69\% of the dry mass, respectively. Energy costs were ca. $0.76 / 0.71 \mathrm{~kJ} / \mathrm{g}$, if calculated from the biomass, and these values were $2.51 / 2.80 \mathrm{~kJ} / \mathrm{g}$ for the dry mass. The estimated carbon input via dead invertebrate individuals contributed $23.15 \mathrm{~g}$ fresh mass (which represented $0.74 \%$ of the total fresh mass), $11.18 \mathrm{~g}(1.18 \%)$ dry mass and $5.59 \mathrm{~g} \mathrm{C}(1.18 \%)$ in the caves. All the cadavers were infected by entomopathogenic fungi. Carbon lost via respiration was $73.71 \mathrm{~g}$, i. e., $2.34 \%$ of the fresh, and $7.75 \%$ of the dry mass at the beginning of overwintering. The total assemblage of the 11 species at the beginning and end of overwintering did not differ significantly in fresh mass $(\mathrm{t}=1.17 ; \mathrm{p}=0.245 ; \mathrm{df}=253)$, but did differ significantly in dry mass $(t=2.28 ; \mathrm{p}=0.023 ; \mathrm{df}=253)$ and energy content $(\mathrm{t}=2.34 ; \mathrm{p}=0.020 ; \mathrm{df}=253)$. The relative importance of species with respect to their abundance, fresh and dry mass and energy bulk is presented in Tab. 6 .
Excrement of both snail species, the two Troglophilus-and some other non-troglobionts, like Diplopoda and Collembola-were relatively abundant close behind the entrance, but were very rare deeper within the caves. In the warm half of the year, e. g., T. neglectus defecated during their daily sojourn up to a few meters behind the entrance, contributing nothing to the troglobiotic community. On the other hand, before overwintering, both Troglophilus defecated; their scarce fecal pellets (about two per 100 cave crickets) were occasionally found deeper inside caves at the overwintering sites, and were consumed by troglobiotic Collembola, Diplopoda and leiodid Coleoptera. These also attracted Pseudoscorpiones, Diplura and carabid Coleoptera. These pellets contributed to the troglobiotic community once a year, but their total mass in all the caves was estimated at less than $200 \mathrm{mg} /$ year. 
Tab. 3: Individual dry mass in the 11 species under investigation. $t$-test between the series at the beginning and at the end of overwintering. Signficant p-values are in bold.

\begin{tabular}{|c|c|c|c|c|c|}
\hline $\begin{array}{c}\text { Species } \\
N \text { of individuals; } \\
\text { beginning-end } \\
\text { of overwintering }\end{array}$ & $\begin{array}{c}\text { Beginning } \\
\text { Mean } \pm S D \\
\text { Min-Max } \\
{[\mathrm{mg}]}\end{array}$ & $\begin{array}{c}\text { End } \\
\text { Mean } \pm S D \\
\text { Min-Max } \\
{[\mathrm{mg}]}\end{array}$ & $\begin{array}{c}\text { Average } \\
\text { difference } \\
\text { beginning-end } \\
\text { of over-wintering [\%] }\end{array}$ & $t ; p$ & $d f$ \\
\hline $\begin{array}{c}\text { F. illyrica } \\
5-5 \\
\end{array}$ & $\begin{array}{l}241.7 \pm 34.7 \\
218.6-292.2 \\
\end{array}$ & $\begin{array}{l}298.1 \pm 22.2 \\
276.3-27.0\end{array}$ & 23.33 & $\begin{array}{c}3.58 \\
\mathbf{0 . 0 0 7}\end{array}$ & 8 \\
\hline $\begin{array}{c}\text { C. lefeburiana } \\
5-6\end{array}$ & $\begin{array}{c}328.7 \pm 35.1 \\
278.4-364.3 \\
\end{array}$ & $\begin{array}{c}218.2 \pm 40.0 \\
172.9-282.9 \\
\end{array}$ & -33.62 & $\begin{array}{l}4.53 \\
\mathbf{0 . 0 0 1}\end{array}$ & 9 \\
\hline $\begin{array}{l}\text { A. aurantiacus } \\
5-5\end{array}$ & $\begin{array}{l}6.5 \pm 0.7 \\
5.3-7.2\end{array}$ & $\begin{array}{l}4.5 \pm 0.4 \\
4.1-5.1\end{array}$ & -30.77 & $\begin{array}{c}5.18 \\
\mathbf{0 . 0 0 1}\end{array}$ & 8 \\
\hline $\begin{array}{l}\text { A. aurantiacus } q \\
5-5\end{array}$ & $\begin{array}{l}8.9 \pm 1.3 \\
7.3-10.9 \\
\end{array}$ & $\begin{array}{l}6.0 \pm 0.9 \\
5.1-7.3 \\
\end{array}$ & -32.58 & $\begin{array}{c}4.11 \\
\mathbf{0 . 0 0 3}\end{array}$ & 8 \\
\hline $\begin{array}{c}\text { G. annulatus } \\
10-9\end{array}$ & $\begin{array}{c}7.91 \pm 2.32 \\
5.6-13.2\end{array}$ & $\begin{array}{c}7.42 \pm 1.02 \\
6.2-9.1\end{array}$ & -6.19 & $\begin{array}{c}0.58 \\
0.669\end{array}$ & 17 \\
\hline $\begin{array}{c}\text { G. titanus } \\
21-24\end{array}$ & $\begin{array}{l}12.6 \pm 1.8 \\
9.7-15.8 \\
\end{array}$ & $\begin{array}{l}13.1 \pm 4.3 \\
7.8-24.6 \\
\end{array}$ & 3.97 & $\begin{array}{l}0.07 \\
0.943 \\
\end{array}$ & 43 \\
\hline $\begin{array}{l}\text { M. menardi } \\
10-12\end{array}$ & $\begin{array}{l}109.7 \pm 52.8 \\
54.8-220.6\end{array}$ & $\begin{array}{l}109.3 \pm 46.6 \\
49.5-194.7\end{array}$ & -0.36 & $\begin{array}{l}0.04 \\
0.965\end{array}$ & 20 \\
\hline $\begin{array}{c}\text { L. schreibersii } \\
5-10\end{array}$ & $\begin{array}{c}24.8 \pm 9.6 \\
16.5-38.0 \\
\end{array}$ & $\begin{array}{c}21.3 \pm 5.6 \\
13.1-28.3 \\
\end{array}$ & -14.11 & $\begin{array}{l}0.75 \\
0.466 \\
\end{array}$ & 13 \\
\hline $\begin{array}{c}\text { T. dubitata } \\
10-11\end{array}$ & $\begin{array}{c}26.4 \pm 3.7 \\
21.0-32.7 \\
\end{array}$ & $\begin{array}{c}22.3 \pm 4.4 \\
16.3-29.2 \\
\end{array}$ & -15.53 & $\begin{array}{c}0.37 \\
\mathbf{0 . 0 2 8}\end{array}$ & 19 \\
\hline $\begin{array}{c}\text { S. libatrix } \\
10-10\end{array}$ & $\begin{array}{c}144.1 \pm 18.2 \\
107.3-165.3 \\
\end{array}$ & $\begin{array}{l}121.1 \pm 26.3 \\
74.5-155.6\end{array}$ & -15.96 & $\begin{array}{l}2.21 \\
\mathbf{0 . 0 4 1}\end{array}$ & 18 \\
\hline $\begin{array}{c}\text { T. cavicola } \\
6-5\end{array}$ & $\begin{array}{c}165.7 \pm 29.6 \\
122.2-200.7\end{array}$ & $\begin{array}{c}135.4 \pm 6.9 \\
125.8-143.6\end{array}$ & -18.29 & $\begin{array}{l}2.16 \\
0.059\end{array}$ & 9 \\
\hline $\begin{array}{c}\text { T. cavicola } \text { ㅇ } \\
5-5\end{array}$ & $\begin{array}{c}172.2 \pm 17.5 \\
154.9-201.7 \\
\end{array}$ & $\begin{array}{c}148.6 \pm 11.6 \\
129.8-160.4 \\
\end{array}$ & -13.7 & $\begin{array}{l}2.58 \\
\mathbf{0 . 0 3 2}\end{array}$ & 8 \\
\hline $\begin{array}{c}\text { T. cavicola I. } \\
10-10\end{array}$ & $\begin{array}{c}31.4 \pm 2.9 \\
26.9-34.5 \\
\end{array}$ & $\begin{array}{l}42.0 \pm 11.7 \\
28.6-52.4 \\
\end{array}$ & 33.76 & $\begin{array}{c}4.65 \\
<\mathbf{0 . 0 0 1} \\
\end{array}$ & 18 \\
\hline $\begin{array}{c}\text { T. neglectus ð } \\
4-2\end{array}$ & $\begin{array}{r}143.6 \pm 37.4 \\
101.7-179.2 \\
\end{array}$ & $\begin{array}{l}112.8 \pm 56.0 \\
73.2-152.4 \\
\end{array}$ & -21.45 & $\begin{array}{l}0.93 \\
0.407 \\
\end{array}$ & 4 \\
\hline $\begin{array}{c}\text { T. neglectus } \text { O } \\
5-6\end{array}$ & $\begin{array}{c}201.2 \pm 29.1 \\
174-234.7 \\
\end{array}$ & $\begin{array}{c}90.6 \pm 42.2 \\
21.0-152.5 \\
\end{array}$ & -54.97 & $\begin{array}{l}2.99 \\
\mathbf{0 . 0 1 5}\end{array}$ & 9 \\
\hline $\begin{array}{c}\text { T. neglectus I. } \\
13-14\end{array}$ & $\begin{array}{c}45.6 \pm 34.0 \\
15.1-121\end{array}$ & $\begin{array}{c}36.8 \pm 30.5 \\
17.8-107.0\end{array}$ & -19.3 & $\begin{array}{l}0.91 \\
0.371\end{array}$ & 25 \\
\hline
\end{tabular}

Tab. 4: Estimated average individual energy budgets for the 11 species under investigation at the beginning and at the end of overwintering. ${ }^{*}$ According to Novak \& Kuštor (1982), ${ }^{1}$ estimated mean budget for arthropods, ${ }^{2}$ provisional budget.

\begin{tabular}{l|c|c|c|c}
\hline Species & $\begin{array}{c}\text { E/g dry mass } \\
{[\mathrm{J} / \mathrm{g}]^{*}}\end{array}$ & $\begin{array}{c}\text { Beginning [J/ } \\
\text { indiv.] }\end{array}$ & $\begin{array}{c}\text { End } \\
{[\mathrm{J} / \text { indiv.] }}\end{array}$ & $\begin{array}{c}\text { Average difference beginning-end } \\
\text { of over-wintering [\%] }\end{array}$ \\
\hline F. illyrica & $20000^{2}$ & 4752.0 & 5962.0 & 25.46 \\
\hline C. lefeburiana & $20000^{2}$ & 6573.2 & 4364.0 & -33.61 \\
\hline A. aurantiacus & $25000^{1}$ & 193.0 & 132.3 & -31.45 \\
\hline G. annulatus & $25000^{1}$ & 257.5 & 173.6 & -32.58 \\
\hline G. titanus & $25000^{1}$ & 315.4 & 328.2 & 4.06 \\
\hline M. menardi & 26449 & 2901.5 & 2890.4 & -0.38 \\
\hline L. schreibersii & 23483 & 530.1 & 550.5 & 3.85 \\
\hline T. dubitata & 25554 & 675.1 & 564.1 & -16.44 \\
\hline S. libatrix & 27127 & 3909.8 & 3284.5 & -15.99 \\
\hline T. cavicola 0 & 24478 & 4054.8 & 3314.3 & -18.26 \\
\hline T. cavicola q & 24579 & 4233.0 & 3651.5 & -13.74 \\
\hline T. cavicola larvae & 23495 & 707.7 & 1006.1 & 42.16 \\
\hline T. neglectus $\delta$ & 21348 & 3065.6 & 2408.1 & -21.45 \\
\hline T. neglectus D & 22967 & 4621.0 & 2081.2 & -54.96 \\
\hline T. neglectus larvae & 21118 & 962.2 & 776.4 & -19.31 \\
\hline
\end{tabular}


Tab. 5: Mean abundance of individuals $(N)$, total fresh and dry mass at the beginning of overwintering, and method of overwintering in the 11 invertebrate species: a active hypogean ecophase, $d$ diapause, q quiescence.

\begin{tabular}{|c|c|c|c|c|c|c|c|c|c|c|}
\hline \multirow[b]{2}{*}{ Species } & \multirow[b]{2}{*}{$\begin{array}{l}N \text { of indiv. } \\
\text { in } 54 \text { caves }\end{array}$} & \multirow[b]{2}{*}{$\begin{array}{l}\text { Me- } \\
\text { thod }\end{array}$} & \multicolumn{3}{|c|}{ Total fresh mass } & \multicolumn{3}{|c|}{ Total dry mass } & \multirow{2}{*}{$\begin{array}{r}\text { Nof } \\
\text { dead } \\
\text { indiv. }\end{array}$} & \multirow{2}{*}{$\begin{array}{r}\text { Dry } \\
\text { necro- } \\
\text { mass }[g]\end{array}$} \\
\hline & & & $\begin{array}{r}\text { Beginning } \\
{[g]}\end{array}$ & End $[g]$ & $\begin{array}{r}\text { Difference } \\
{[\%]}\end{array}$ & $\begin{array}{r}\text { Beginning } \\
{[\mathrm{g}]}\end{array}$ & End $[g]$ & $\begin{array}{r}\text { Difference } \\
{[\%]}\end{array}$ & & \\
\hline F. illyrica & 333 & $q$ & 647.45 & 556.94 & -13.98 & 79.12 & 99.27 & 25.47 & 0 & 0.00 \\
\hline C. lefeburiana & 12 & $q$ & 26.43 & 18.99 & -28.15 & 3.94 & 2.62 & -33.50 & 0 & 0.00 \\
\hline A. aurantiacus & 8406 & $d$ & 147.19 & 161.14 & 9.48 & 64.89 & 44.47 & -31.47 & 430 & 3.31 \\
\hline G. annulatus & 86 & $q$ & 2.57 & 2.64 & 2.72 & 0.89 & 0.60 & -32.58 & 0 & 0.00 \\
\hline G. titanus & 109 & $q$ & 4.68 & 4.88 & 4.27 & 1.38 & 1.43 & 3.62 & 10 & 0.08 \\
\hline M. menardi & 772 & $a$ & 191.60 & 224.34 & 17.09 & 84.69 & 79.66 & -5.94 & 10 & 1.10 \\
\hline L. schreibersii & 147 & $d$ & 8.42 & 10.31 & 22.45 & 3.32 & 3.45 & 3.92 & 1 & 0.02 \\
\hline T. dubitata & 210 & $d$ & 12.35 & 10.58 & -14.33 & 5.55 & 4.64 & -16.40 & 20 & 0.53 \\
\hline S. libatrix & 309 & $q$ & 80.69 & 70.71 & -12.37 & 44.54 & 37.41 & -16.01 & 40 & 5.76 \\
\hline T. cavicola $\hat{o}$ & 874 & d & 417.57 & 520.96 & 24.76 & 144.78 & 118.34 & -18.26 & 0 & 0.00 \\
\hline T. cavicola + & 1102 & $d$ & 596.42 & 674.12 & 13.03 & 189.79 & 163.71 & -13.74 & 0 & 0.00 \\
\hline T. cavicola larvae & 3772 & $q$ & 328.43 & 563.20 & 71.48 & 113.61 & 161.52 & 42.17 & 3 & 0.09 \\
\hline T. cavicola $\Sigma$ & 5748 & & 1342.42 & 1758.28 & 30.98 & 448.18 & 443.57 & -1.03 & & 0.09 \\
\hline T. neglectus ठ & 377 & q & 190.28 & 166.82 & -12.33 & 54.14 & 42.53 & -21.44 & 1 & 0.14 \\
\hline T. neglectus 9 & 476 & $q$ & 309.05 & 166.99 & -45.97 & 95.77 & 43.13 & -54.97 & 0 & 0.00 \\
\hline T. neglectus larvae & 1373 & $q$ & 182.98 & 204.64 & 11.84 & 62.56 & 50.48 & -19.31 & 2 & 0.09 \\
\hline T. neglectus $\Sigma$ & 2226 & & 682.31 & 538.45 & -21.08 & 212.47 & 136.14 & -35.93 & & 0.23 \\
\hline Total & & & 3146.11 & 3357.26 & 6.71 & 948.97 & 853.26 & -10.09 & & 11.18 \\
\hline
\end{tabular}

Tab. 6: The \% contribution of species among the 11 species to total number of species, fresh and dry mass, carbon and energy in caves in winter.

\begin{tabular}{l|r|r|l|l|l|r|r}
\hline $\boldsymbol{N}$ & & \multicolumn{2}{|l|}{ Fresh mass } & \multicolumn{2}{l|}{ Dry mass-Carbon } & \multicolumn{2}{l}{ Energy } \\
\hline A. aurantiacus & 45.79 & T. cavicola & 42.65 & T. cavicola & 47.16 & T. cavicola & 48.15 \\
\hline T. cavicola & 31.31 & T. neglectus & 21.68 & T. neglectus & 22.36 & T. neglectus & 20.70 \\
\hline T. neglectus & 12.13 & F. illyrica & 20.61 & M. menardi & 8.91 & M. menardi & 9.92 \\
\hline M. menardi & 4.21 & M. menardi & 6.09 & F. illyrica & 8.47 & A. aurantiacus & 7.18 \\
\hline F. illyrica & 1.81 & A. aurantiacus & 4.68 & A. aurantiacus & 6.83 & F. illyrica & 7.13 \\
\hline S. libatrix & 1.68 & S. libatrix & 2.56 & S. libatrix & 4.69 & S. libatrix & 5.35 \\
\hline T. dubitata & 1.14 & C. lefeburiana & 0.84 & T. dubitata & 0.58 & T. dubitata & 0.63 \\
\hline L. schreibersii & 0.80 & T. dubitata & 0.39 & C. lefeburiana & 0.41 & C. lefeburiana & 0.35 \\
\hline G. titanus & 0.59 & L. schreibersii & 0.27 & L. schreibersii & 0.35 & L. schreibersii & 0.34 \\
\hline G. annulatus & 0.47 & G. titanus & 0.15 & G. titanus & 0.15 & G. titanus & 0.15 \\
\hline C. lefeburiana & 0.07 & G. annulatus & 0.09 & G. annulatus & 0.09 & G. annulatus & 0.10 \\
\hline
\end{tabular}

\section{DISCUSSION}

Terrestrial fauna migrating between surface and hypogean habitats-i. e., the non-troglobiotic taxa-have been considered among the most important vectors of carbon supplying subterranean communities (Culver \& Pipan 2009; Fong 2011). In contrast to this general logical appearance, this statement should be carefully examined in every karstic region settled by distinctive fauna, as we demonstrate in this contribution for Central European caves.

Despite ambiguous data in some species and stages under study, which deserve renewed measurement, we can identify the general trends for non-troglobiotic animals from caves in Slovenia during winter. The total biomass of non-troglobiotic taxa increases slightly during winter, while the dry mass decreases, indicating that utilization of reserve lipids is the most important metabolic process in these species overwintering in central European caves (Lipovšek et al. 2004, 2008, 2009, 2011; Novak et al. 2004). The more frequent species and those with grater biomass share the first five places in a ranking of their importance to subterranean habitats in either abundance, fresh and dry mass or energy bulk, compris- 
ing $93.08-95.71 \%$ ratios among the 11 species. These are T. cavicola, T. neglectus, M. menardi, F. illyrica and A. aurantiacus. While $M$. menardi (mostly, see the life cycle in Novak et al. 2010) and L. schreibersii are residents of subterranean habitats, the migratory species alternate between epigean and hypogean habitats, with minimal total loss $(0.74 \%)$ of their biomass in caves during winter. Besides, all the individuals that die in caves are infected by entomopathogenic fungi and are thus not directly available to subterranean animals, at least not to arthropods, which strictly avoid contact with infested cadavers. Predated individuals (Novak et al. 2010) and the scanty excrement produced during pre-overwintering defecation represent the only resource of non-troglobiotic taxa in Slovenian caves. The overall amount of these contributions does not exceed $1 \%$ of their biomass, i. e., about $0.3 \%$ dry mass and $0.15 \%$ organic carbon. In contrast to the general assumption and our hypothesis, in Central European caves, non-troglobiotic taxa contribute very little to the deep troglobiotic species. Additionally, predated individuals contributed maximally $0.15 \% \mathrm{C}$. This low carbon input might be the main reason why a major group of troglobionts inhabit shallow subterranean habitats (Novak et al. 2012).

Many points must be considered in providing a credible estimate of each species contribution to biomass, dry mass, necromass, energy and/or carbon input in hypogean habitats: its life cycle and type of dormancy, preferred hypogean habitats, the biomass bulk of immigrating and emigrating individuals, the duration of its hypogean ecophase, the strategy for creation and utilization of energy-supporting reserve stocks, the time and space frames of specific investigations and the form of species contribution (prey, necromass or excrement) in the subterranean habitat. In our study, species abundance was underestimated by at least $30 \%$ of the visible population (Novak, unpublished). While more accurate data of this kind would improve our quantitative estimates of mass, energy and carbon flow, they would not change the outcomes on the importance of migratory non-troglobionts to troglobiotic communities.

Our results represent actual conditions in a specific period and region. These conditions change from year to year and vary among different regions and caves. In studies on the biomass, energy and/or carbon flow supplied by non-troglobiotic animals to caves it, is of special importance to check whether these individuals or their products, like eggs, are predated, or if they defecate there, or whether their cadavers are consumed by species which are more or less permanently resident in caves.

For analytical purposes, in the context of carbon flux, large invertebrates can be compared with coarse particulate organic carbon, which moves relatively short distances before it is broken down or consumed (Simon \& Benfield 2001). Such a distribution is also true for all the species under study, which are most abundant in the upper $10 \mathrm{~m}$ beneath the surface (Novak et al. 2012). Gibert (1986) found that dissolved organic carbon is the most important among abiotic carbon sources in subterranean water. Analogously, it seems justified to consider the possibility that microfauna are the most significant biotic vector of carbon supply to terrestrial hypogean habitats.

\section{CONCLUSIONS}

Terrestrial invertebrates overwintering in caves have hypothetically been considered an important vector conveying carbon from the epigean to the hypogean habitat, but in reality they represent a rather limited food resource for hypogean species. In Central European caves, five among the 11 species contribute $93.08-95.71 \%$ ratios of the total abundance, biomass and dry mass, energy and incorporated carbon of the 11 species. These five species migrate between the epigean and hypogean habitats and are the most important ones in this respect. Despite this, they contribute a negligible amount of carbon to subterranean fauna.

\section{ACKNOWLEDGEMENTS}

We are indebted to David Culver and two anonymous referees for their insightful comments and corrections of the initial version of the manuscript, and to Michelle Gadpaille for valuable improvements to the language. This study was partly supported by the Slovene Research Agency within the research program Biodiversity (grant P1-0078). 


\section{REFERENCES}

Culver, D.C. \& T. Pipan 2009: Biology of Caves and Other Subterranean Habitats.- Oxford University Press, Oxford, U.K., pp. 254.

Deharveng, L. \& A. Bedos, 2000: The cave fauna of southeast Asia. Origin, evolution and ecology. In: Wilkens, H., D.C. Culver \& W.F. Humpreys (Eds.): Subterranean ecosystems. Elsevier, pp. 603-632.

Denlinger, D.L., 2002: Regulation of diapause.- Annual Review of Entomology 47, 93-122.

Engel. A.S., 2005: Observations on the biodiversity of sulfidic karst habitats.- Journal of Cave and Karst Studies, 69, 187-206.

Fong, D.W., 2011: Management of subterranean fauna in karst.- In: van Beynen, P.E. (Ed.), Karst Management. Springer, pp. 201-224.

Gibert, J., 1986: Écologie d'un système karstique jurassien. Hydrogéologie, dérive animale, transits de matières, dynamique de la population de Niphargus (Crustace' Amphipode).- Mémoires de Biospéologie, 13, 1-379.

Gnaspini, P., 2005: Guano community. In: Encyclopedia of Caves. White, W.B. \&. D.C. Culver (Eds.). Springer, pp. 201-364.

Houghton, R.A., F. Hall \& S.J. Goetz, 2009: Importance of biomass in the global carbon cycle.Journal of Geophysics Research 114, G00E03, doi:10.1029/2009JG000935.

Jequier, J.-P., 1964: Étude écologique et statistique de la faune terrestre d'une caverne du Jura Suisse au cours d'une année d’observations.- Revue Suisse de Zoologie, 71, 13-370.

Lavoie, K.H., K.L. Helf \& T.L. Poulson, 2007: The biology and ecology of North American cave crickets.- Journal of Cave and Karst Studies, 69, 114-134.

Lipovšek Delakorda, S., I. Letofsky-Pabst, T. Novak, M. Giovanelli, F. Hofer \& M.A. Pabst, 2008: Application of elemental microanalysis to elucidate the role of spherites in the digestive gland of the helicid snail Chilostoma lefeburiana.- Journal of Microscopy, 231, 38-46. DOI: 10.1111/j.1365-2818.2008.02015.x

Lipovšek Delakorda, S., T. Novak, F. Janžekovič, L. Senčič \& M.A. Pabst, 2004: A contribution to the functional morphology of the midgut gland in phalangiid harvestmen Gyas annulatus and Gyas titanus during their life cycle.- Tissue \& Cell, 36, 275-282. doi:10.1016/j.tice.2004.04.003
Lipovšek, S., I. Letofsky-Pabst, T. Novak, F. Hofer \& M.A. Pabst, 2009: Structure of the Malpighian tubule cells and annual changes in the structure and chemical composition of their spherites in the cave cricket Troglophilus neglectus Krauss, 1878 (Rhaphidophoridae, Saltatoria).- Arthropod Structure and Development 38, 315-327. doi:10.1016/j. asd.2009.02.001

Lipovšek, S., T. Novak, F. Janžekovič \& M.A. Pabst, 2011: Role of the fat body in the cave crickets Troglophilus cavicola and Troglophilus neglectus (Rhaphidophoridae, Saltatoria) during overwintering.- Arthropod Structure and Development, 40, 54-63. doi:10.1016/j.asd.2010.09.002

Novak, T., 2005: Terrestrial fauna from cavities in Northern and Central Slovenia, and a review of systematically ecologically investigated cavities.- Acta Carsologica, 34, 169-210.

Novak, T. \& V. Kuštor, 1982: Contribution a la connaissance de la biomasse et du bilan énergetique de la faune des entrées de grotte en Slovenie (Yougoslavie).- Mémoires de Biospéologie, 8, 82-89.

Novak, T. \& V. Kuštor, 1983: On Troglophilus (Rhaphidophoridae, Saltatoria) from North Slovenia (YU). Mémoires de Biospéologie, 10, 127-137.

Novak, T., S. Lipovšek Delakorda, L. Senčič, M.A. Pabst \& F. Janžekovič, 2004: Adaptations in phalangiid harvestmen Gyas annulatus and G. titanus to their preferred water current adjacent habitats. Acta Oecologica, 26, 45-53.

Novak, T., M. Perc, S. Lipovšek \& F. Janžekovič, 2012: Duality of terrestrial subterranean fauna. International Journal of Speleology, 41, 181-188.

Novak, T., T. Tkavc, M. Kuntner, A.E. Arnett, S. Lipovšek Delakorda, M. Perc \& F. Janžekovič, 2010: Niche partitioning in orbweaving spiders Meta menardi and Metellina merianae (Tetragnathidae).- Acta Oecologica, 36, 522-529.

Perruchoud, D., L. Walthert, S. Zimmermann \& P. Lüscher, 2000: Contemporary carbon stocks of mineral forest soils in the Swiss Alps.- Biogeochemistry, 50, 111-136.

Por, F.D., 2007: Ophel: a groundwater biome based on chemoautotrophic resources. The global significance of the Ayyalon cave finds, Israel.- Hydrobiologia, 592, 1-10.

Riebeek, H, 2011: The carbon cycle. - http://earthobservatory.nasa.gov/Features/CarbonCycle/ 
Salonen, K., J. Sarvala, I. Hakala \& M.-L. Viljanen, 1976: The relation of energy and organic carbon in aquatic invertebrates.- Limnology and Oceanography, 21, 724-730.

Sârbu, S.M., T.C. Kane \& B.K. Kinkle, 1996: A chemoautotrophically based cave ecosystem.- Science, 272, 1953-1955.

Schmidt, M.W.I., M.S.Torn, S. Abiven, T. Dittmar, G. Guggenberger, I.A. Janssens, M. Kleber, I. KögelKnabner, J. Lehmann, D.A.C. Manning, P. Nannipieri, D.P. Rasse, S. Weiner \& S.E. Trumbore, 2011: Persistence of soil organic matter as an ecosystem property.- Nature, 478, 49-56

Simon, K.S. \& E.F. Benfield, 2001: Leaf and wood breakdown in cave streams.- Journal of the North American Benthological Society, 482, 31-39.

Simon, K.S. \& E.F. Benfield, 2002: Ammonium retention and whole stream metabolism in cave streams.- Hydrobiologia, 482, 31-39.
Simon, K.S., T. Pipan \& D.C. Culver, 2007: A conceptual model of the flow and distribution of organic carbon in caves.- Journal of Cave and Karst Studies, 69, 279-284.

Taylor, S.J., J. Krejca \& M.L. Denight, 2005: Foraging and range habitat use of Ceuthophilus secretus (Orthoptera Rhaphidophoridae), a key trogloxene in central Texas cave communities.- American Midland Naturalist, 154, 97-114.

Tercafs, R.R., 2001: The protection of the subterranean environment. Conservation principles and management tools.- P.S. Publishers, Luxembourg, $400 \mathrm{p}$.

U.S.DOE, 2008: Carbon cycling and biosequestration: Integrating Biology and climate through system science.- Report from the March 2008 Workshop, Doe/SC-108, U.S. Deptartment of Energy Office of Science, $133 \mathrm{p}$. 